\title{
Spontaneous Emission Control with Planar Dielectric Structures: An Asset for Ultrasensitive Fluorescence Analysis
}

\author{
Cédric Begon ${ }^{\text {a) }}$, Hervé Rigneault ${ }^{\text {a) }}$, Per Jonsson ${ }^{\text {b) }}$ and John G. Rarity ${ }^{\text {c) }}$
}

a) Institut Fresnel, Ecole Nationale Supérieure de Physique de Marseille, Marseille, France

${ }^{b)}$ KTH-Electrum, Department of Electronics, Kista, Sweden

${ }^{c}$ Defence Evaluation and Research Agency, Malvern, United Kingdom

\section{Correspondence to}

Cédric Begon

Institut Fresnel

Ecole Nationale Supérieure de Physique de Marseille

Domaine universitaire de St Jérôme

13397 Marseille cedex 20, France

phone +33491288327

fax +33491288067

e-mail cedric.begon@enspm.u-3mrs.fr

submitted 11 May 2000

published 30 Aug 2000

\section{Introduction}

The success of ultrasensitive analysis of fluorescent molecules in solution or on surfaces at ambient temperature has been built on two major experimental requirements [1]. First, optimizing the fluorescence collection efficiency of the apparatus, and secondly, discriminating as much as possible the relevant fluorescence signal against background. Great efforts have been devoted to the second point in order to achieve a sensitivity down to the single molecule level. In this respect, two spatial selection principles have been successfully applied in addition to traditional spectral filters to reduce the contribution from surrounding materials (mostly Rayleigh and Raman scattering). The first is the far-field confocal microscopy set-up which allows one to obtain a collection volume in solution as small as $0.2 \mathrm{fl}$ with high numerical aperture (NA) microscope objectives [2, 3]. Even more selective is the method of near field scanning optical microscopy (NSOM), which defines sub-diffraction limit exciting spots on surfaces [4, 5]. In both cases, fluorescence is usually collected through a high NA microscope objective and the collection reaches about 30\% of the total emitted light (1.2 NA, water immersion). The goal of this paper is to show that this value is not a limit and is likely to be notably increased by treating the problem at the very root of the spontaneous emission phenomenon.

Spontaneous emission is often presented in the frame of quantum electrodynamics as stimulated emission, stimulated by vacuum field fluctuations. As a result, it is not an immutable property of the emitter, and Purcell first pointed out in 1946 that the confinement of an emitter in a cavity whose dimensions would be of the order of the emission wavelength should alter the spontaneous emission rate from its value in free space [6]. This possibility is summarised in Fermi's golden rule which shows that the spontaneous emission rate of an electric dipole transition (the inverse of its spontaneous lifetime) 
depends both on the local vacuum electric field amplitude and on the density of electromagnetic modes at the dipole location. The modification of electromagnetic boundaries in the vicinity of the source not only makes its radiation pattern non-isotropic but possibly induces changes of radiative lifetime.

After the seminal experiment of Drexhage which demonstrated spontaneous emission control with a single mirror in the visible range [7], striking results of spontaneous emission inhibition or enhancement were obtained in the microwave domain with Fabry-Perot cavities which modify both the mode density and the vacuum field intensity [8, 9]. They initiated the very productive field of cavity quantum electrodynamics (CQED), and spontaneous emission control was thoroughly investigated with the almost ideal systems of atomic physics. The interest soon spread to the solid state community where spontaneous emission control appeared as a remarkable tool to increase the brightness of LEDs and make efficient monolithic microlasers. The progress in microfabrication techniques permitted the study of a wide variety of microcavity geometries focussing on their ability to control spontaneous emission and the different coupling regimes which can exist depending on the oscillator strength of the emitter [10].

With the existing structures, it is clear that notable lifetime modifications are only accessible for sources with narrow emission spectra, such as quantum boxes [11]. Nevertheless, with large spectrum emitters like Lanthanide ions in amorphous materials or organic dyes, it is still possible to use microcavities to tailor the radiation pattern and concentrate the emitted light in specific directions. This is therefore particularly attractive with a view to increasing fluorescence collection efficiency in biological ultra-sensitive analysis. In this context, the microstructure has to be robust enough in its principle to work on large wavelength domains and with sufficiently low tuning requirements to handle emitters in liquid phase.

We present in the following section the assets of planar dielectric structures to fulfill those points. Then, two experimental realisations are discussed, a mirror and a microcavity, both used in confocal FCS apparatus to control the emission of a small number of molecules of Cyanine 5 and Rhodamine 6G.

\section{Theoretical Model and Preliminary Results}

Stacks of alternate high- and low-index thin dielectric layers are commonly used to make interference filters and highly reflecting mirrors (also known as DBRs, Distributed Bragg Reflectors). Two properties of those planar thin film stacks are particularly interesting: depending on the wavelength range of interest, appropriate dielectric materials show extremely low absorption coefficients. Secondly, the spectral characteristics of any given stack can be very accurately monitored during the deposition process, layer by layer. It is therefore possible to build Fabry-Perot type microstructures whose resonance matches the emission wavelength of the source $\lambda_{0}$ [12]. This is the case if the optical thickness of the spacing layer between the two parallel mirrors is an integer multiple of $\lambda_{0} / 2$. One has to place the source at an anti-node of the resonant electric field inside the cavity to obtain the maximum emission in this mode [13-15].

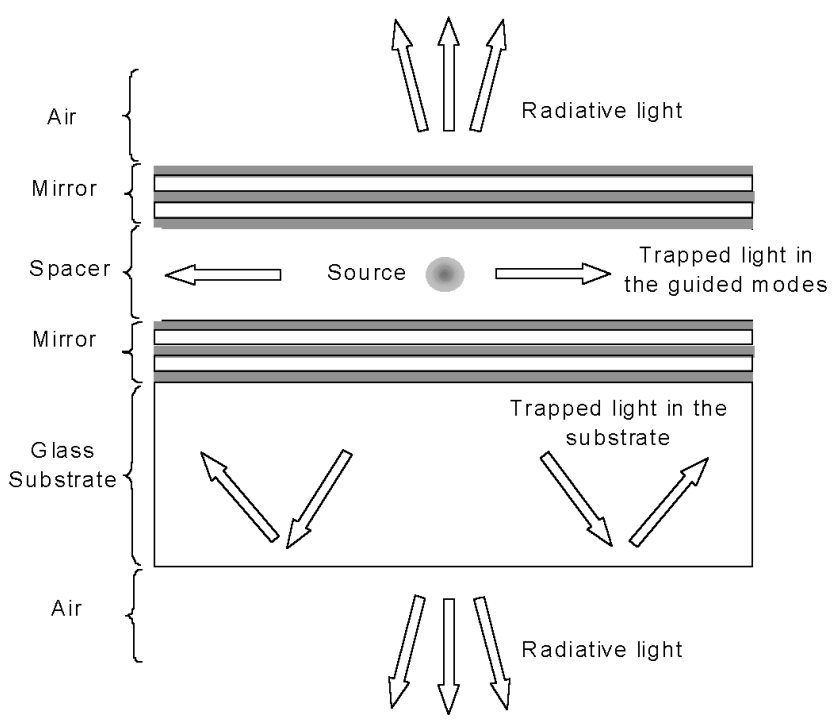

Fig. 1. Radiative and trapped light emitted by a source located inside a planar dielectric microcavity

The emission of a source described as a monochromatic three-dimensional electromagnetic dipole in a planar dielectric microcavity grown on a glass substrate has been theoretically studied in [16], in the weak coupling regime approximation. For each particular position of the source in a dielectric stack, the emission distributed on a complete basis of orthonormal electromagnetic modes can be computed. As a result, the radiation pattern of the source and the ratio of the power extracted in the air on the total emitted power are also accessible. It turns out that even if the emitter is optimally coupled with the resonant mode of the Fabry-Perot cavity, a large amount of light remains trapped in the structure, either in the guided modes or in the substrate, as sketched on figure 1 . This puts a practical limit on the light extraction achievable with planar microcavities [17, 18]. Nevertheless, one can still take advantage of the emission directivity and spectral selection which are classically obtained with planar microcavities.

As a demonstrative example, let us describe briefly a very simple structure whose stack formula is aair-HLH4LHLHsilica substrate\}, where $\mathrm{H}$ and $\mathrm{L}$ stand for high- and lowrefractive index quarter wavelength layers respectively, at $\lambda$ $=620 \mathrm{~nm}$. The corresponding dielectric materials are Zinc sulfide $\left(n_{H}=2.36\right)$ and Cryolite $\left(n_{L}=1.3\right)$. This stack forms 
a microcavity with a spacer of $4 \mathrm{~L}$ optical thickness between two mirrors. The resonance wavelength of $620 \mathrm{~nm}$ is close to the maximum emission of a UV-excitable binuclear $\mathrm{Eu}^{3+}$ chelate, whose spectrum is visible on Figure 2 (the details of the chemical structure are in [19]]. We have chosen this organic complex for its narrow emission band and its large Stokes shift. Our pump wavelength of $337 \mathrm{~nm}$ (pulsed Nitrogen laser), falling outside the stop-bands of the mirrors, the pump beam is not affected by undesirable filtering effects [20].

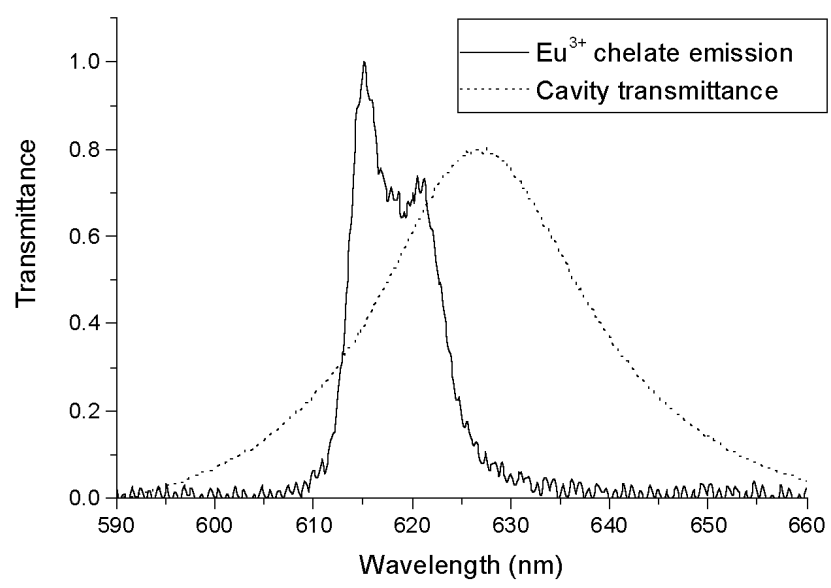

Fig. 2. Normalized emission spectrum of the binuclear Europium chelate (solid line) and transmission peak of the cavity sample air-HLHL-Eu ${ }^{3+}$ chelate-3LHLH-silica substrate air\} (broken line).

Figure 3 shows the result of a calculation based on the modal analysis cited above [16]: the total normalized power emitted in the air by a three-dimensional dipole at $620 \mathrm{~nm}$ as a function of its position in the stack. During the evaporation process, we have incorporated a drop of concentrated chelate solution in methanol at the location of a maximum of this curve. This has shifted the transmission peak to $626 \mathrm{~nm}$, for reasons which are not well understood (details are exposed in [21]). The polar radiation pattern observed at the wavelength of maximum emission, $615 \mathrm{~nm}$, is shown on figure 4. The emission is blue-shifted with respect to the resonance wavelength of the microcavity, therefore it is maximum at an angle of approximately $15^{\circ}$. The agreement with the computed radiation pattern is excellent, in spite of the unexplained shift of the cavity transmission peak. We observe the announced effects of directivity and spectral selection. Furthermore, this simple example illustrates the possibilities of spontaneous emission control offered by a very low quality factor cavity $(Q$ $\left.=\lambda_{0} / \Delta \lambda \sim 25\right)$. The calculation for a 3D-dipole located at the chelate position and emitting at $615 \mathrm{~nm}$ indicates that the amount of power extracted in the air on the coating side reaches $23.6 \%$ of the total emitted light. This extraction takes place essentially in a cone of $30^{\circ}$ half-angle, and hence represents more than three times the result we would obtain in bulk material in the same solid angle.

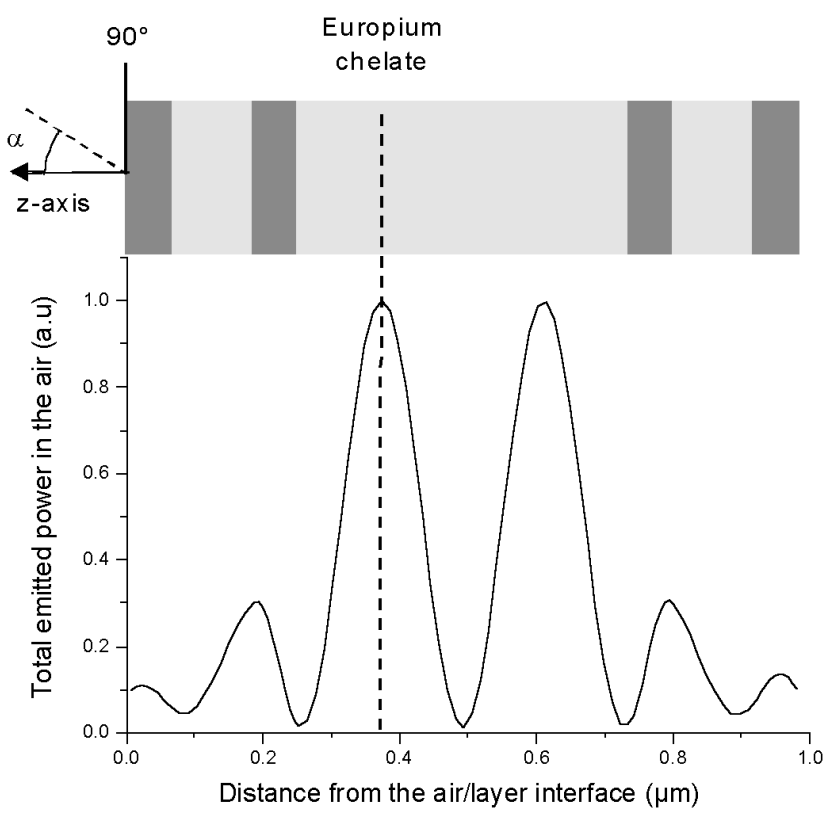

Fig. 3. Normalized power emitted in the air at $620 \mathrm{~nm}$ by a three-dimensional dipole located inside the structure\{airHLH4LHLH-silica substrate-air\} as a function of its position in the stack.

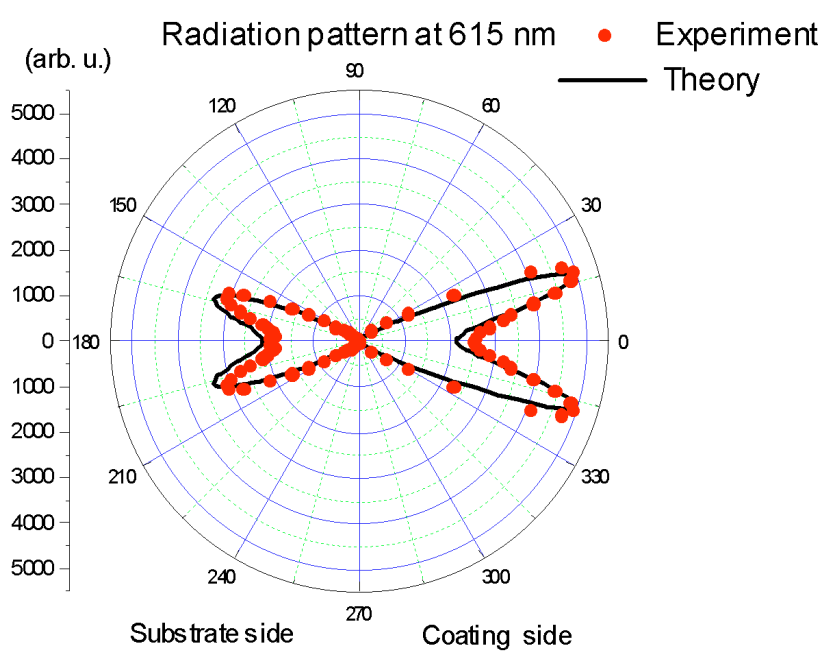

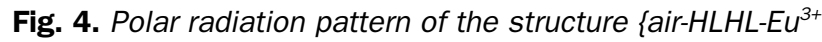
chelate-3LHLH-silica substrate-air\} measured at $615 \mathrm{~nm}$. The excitation wavelength was $337 \mathrm{~nm}$. For each experimental point, the light was collected in a solid angle of $10^{-3}$ steradian. 


\section{Molecules RESEARCH PAPER}

\section{Mirror Experiments}

\section{Methods and Results}

We focus now on the properties of a single dielectric mirror for controlling the spontaneous emission of a spectrally broad emitter in liquid medium: Cyanine 5 in dimethyl formamide (DMF), excited at $633 \mathrm{~nm}$. DMF $\left(\mathrm{n}_{\mathrm{DMF}}=1.43\right)$ has been chosen for its low volatility and low viscosity. We have specially developed a mirror whose reflectivity is high in the Cyanine 5 emission band but low for the pump wavelength, as shown on figure 5 . This transmittance profile has been obtained with the stack formula \{air- $(5 \mathrm{H} 5 \mathrm{~L})^{3} 5 \mathrm{HL}-$ silica substrate\}, where $\mathrm{H}$ and $\mathrm{L}$ stand for a high and low refractive index quarter-wavelength layer respectively, at $680 \mathrm{~nm}$. The dielectric materials are Tantalum pentoxide $\left(\mathrm{n}_{\mathrm{H}}\right.$ $=2.23$ ) and silica $\left(n_{L}=1.5\right)$. They have been evaporated in a Balzer BAP800 plant with the Ion Plating technique, that makes layers resistant enough to endure contact with liquids.

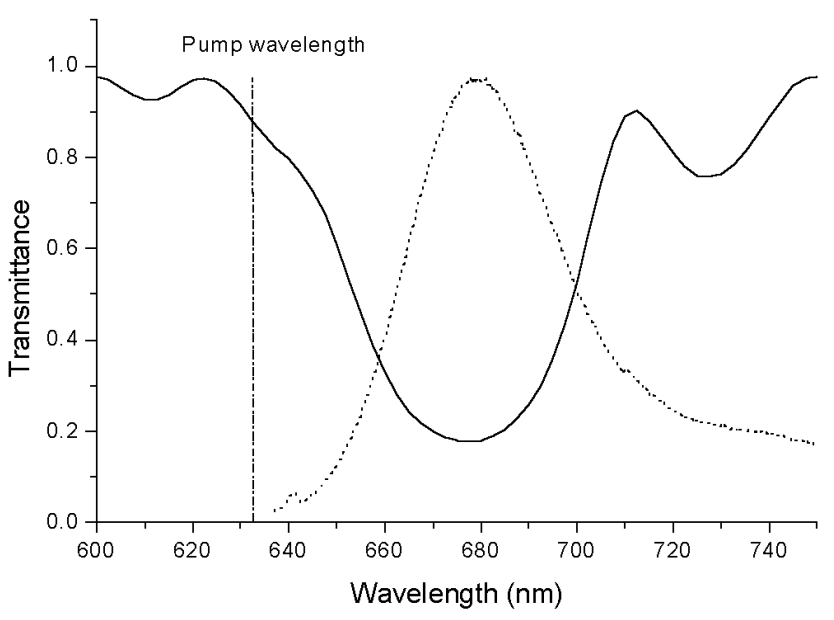

Fig. 5. Transmittance spectrum of the mirror (solid line) and normalized emission spectrum of Cyanine 5 in DMF (broken line).

We want here to assess the detection enhancement which is accessible with such a simple structure in a realistic single molecule detection experiment. We have adopted a confocal fluorescence correlation setup visible on figure 6 . The design is conventional $[2,22,23]$ apart from using long working distance objective (Olympus LMPIanFI, 50x, Olympus, Japan) with 0.5 numerical aperture. The dichroïc and bandpass filters (110 nm bandwidth) are designed and grown in-house to maximize the transmission in the emission range of Cyanine 5 molecules. Our single photon counting device is an avalanche photodiode (SPCM-AQR14, EG\&G Canada) with negligible darkcount (50 counts per second) and $65 \%$ quantum efficiency at $680 \mathrm{~nm}$. We aim at comparing the average count rates we obtain for a molecule lying at the top of our mirror and for a molecule on a bare silica substrate. In both cases, the samples are prepared by pressing a $2 \mu \mathrm{l}$ drop of dilute Cyanine 5 solution in DMF $\left(5.10^{-8} \mathrm{M}\right)$ between the substrate and a $0.2 \mathrm{~mm}$-thick Suprasil silica coverslip (Heraeus, France). Note that we lack accurate control on the liquid layer thickness, and hence on the excited volume. The sample's plane is placed orthogonally to the optical axis. The pump power is about $25 \mathrm{~kW} / \mathrm{cm}^{2}$ and avoids too strong photobleaching effects. The electronics estimates the photocount correlation function

$$
\mathrm{g}^{(2)}(\tau)=\frac{\langle\mathrm{I}(\mathrm{t}) \mathrm{I}(\mathrm{t}+\tau)\rangle}{\langle\mathrm{I}\rangle^{2}}
$$

where $\mathrm{I}(\mathrm{t})$ is the measure intensity at time $\mathrm{t}$ and $\mathrm{I}(\mathrm{t}+\tau)$ the intensity measure a time later.

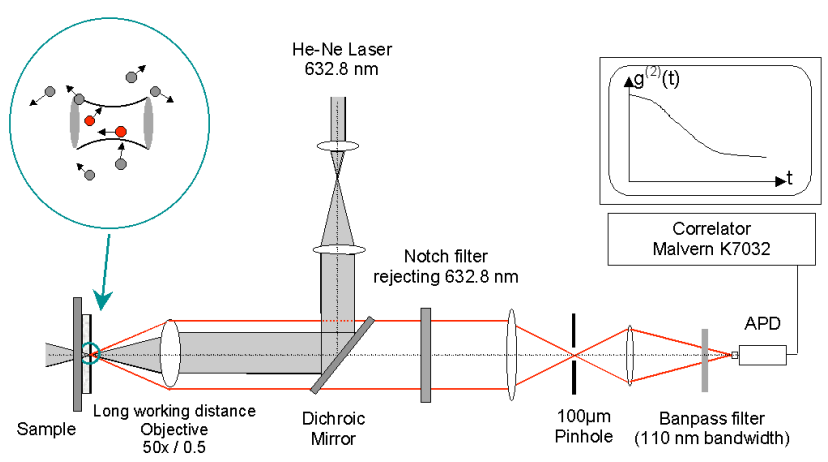

Fig. 6. Confocal fluorescence correlation spectroscopy setup.

Two typical autocorrelation curves are shown in figure 7, one for each sample type. Due to the planar geometry of our sample, we naturally adopt the simple two-dimensional diffusion model to fit the correlation curves, with a correction for the background count rate $\mathrm{I}_{\mathrm{b}}$ which holds in the linear regime $[2,24]$ :

$$
g^{(2)}(\tau)=1+\left(1-\frac{I_{b}}{I}\right)^{2} \frac{1}{M\left(1+\tau / t_{d}\right)}
$$

$M$ stands for the average number of molecules in the observable volume element, and $t_{d}$ is the average residence time in the volume element. $I_{b}$ is the background count rate and $I$ is the total count rate observed in the experiment.

The results we can extract from the curves presented in figure 6 are summarized in Table 1. Despite our excellent rejection at $633 \mathrm{~nm}$ (better than $10^{-8}$ ), the background intensity in the mirror case reaches 5 kilocounts per second, five times the counts obtained with the bare substrate. Note that the reflection coefficient of the mirror at $633 \mathrm{~nm}$ is approximately $11 \%$ for normally incident light but goes up to $70 \%$ for light incident at $30^{\circ}$, the limit angle of our focused pump beam. In addition with laser reflected and scattered light, the proper fluorescence of the dielectric 
layers could possibly contribute to the background. It turns out that the presence of the mirror increases the count rate per molecule averaged over a long period by a factor of 1.3 approximately.

\begin{tabular}{|l|c|c|}
\hline & $\begin{array}{c}\text { Cyanine 5 on bare } \\
\text { substrate }\end{array}$ & Cyanine 5 on mirror \\
\hline $\begin{array}{l}\text { I: average total count rate } \\
\text { (kilo-counts per second) }\end{array}$ & $300 \pm 20$ & $550 \pm 25$ \\
$\begin{array}{l}\mathbf{I}_{\mathbf{b}}: \text { average background } \\
\text { count rate } \\
\text { (kilo-counts per second) }\end{array}$ & $1 \pm 0.2$ & $5 \pm 1$ \\
$\begin{array}{l}\text { M: average number of } \\
\text { molecules }\end{array}$ & $20 \pm 0.2$ & $27.8 \pm 0.3$ \\
\hline $\begin{array}{l}\text { Count rate per molecule on } \\
\text { average } \\
\text { (kilo-counts per second) }\end{array}$ & $15 \pm 1.1$ & $19.6 \pm 1.1$ \\
\hline $\mathbf{t}_{\mathbf{d}}:$ diffusion time $(\mu \mathrm{s})$ & $738 \pm 14$ & $698 \pm 17$ \\
\hline
\end{tabular}

Table 1. Results extracted from the autocorrelation curves shown in figure 7

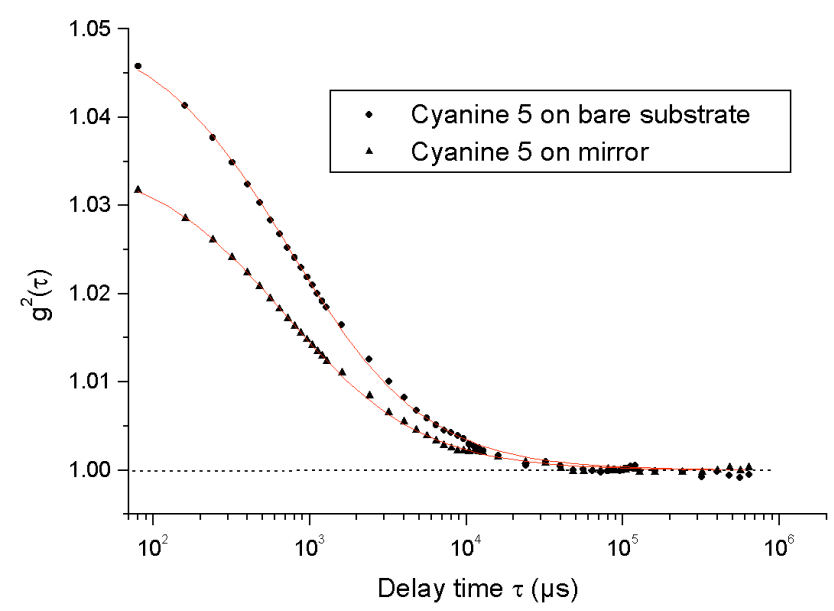

Fig. 7. Typical autocorrelation curves obtained with molecules on the top of the mirror and molecules on a bare silica substrate. The curves are fitted with formula (2).

\section{Discussion}

In contrast with the preliminary example presented in the first section, the emitters are not at a fixed distance from the mirror but are distributed in the liquid layer along the $z$ optical axis. Thus, the measured count rates integrate the contributions from emitters which feel different values of the vacuum electric field. In our case, the sum of all those contributions tends towards a limit which is well estimated by integrating the radiation patterns for all possible emitter positions over a $3 \mu \mathrm{m}$-thickness. The result of the computation for our two samples is shown in figure 8 , for a source emitting at $680 \mathrm{~nm}$. Note that in both cases, the dipoles are assumed to be excited without saturation effects. Let us call $P_{c}$ the power collected in the $30^{\circ}$ acceptance angle of our objective. At $680 \mathrm{~nm}$ the mirror effect multiplies $\mathrm{P}_{\mathrm{c}}$ by 1.4 comparatively to the substrate case. Taking into account the spectral profile of the mirror by integrating $P_{c}$ over the entire Cyanine 5 spectrum gives a slightly inferior factor:1.35.

This theoretical value is in good agreement with the 1.3 ratio of the experimental counting rates per molecule observed in the two cases. It could be certainly increased by designing a mirror with higher and broader reflectivity in the Cyanine emission band, and by collecting light in larger a solid angle.

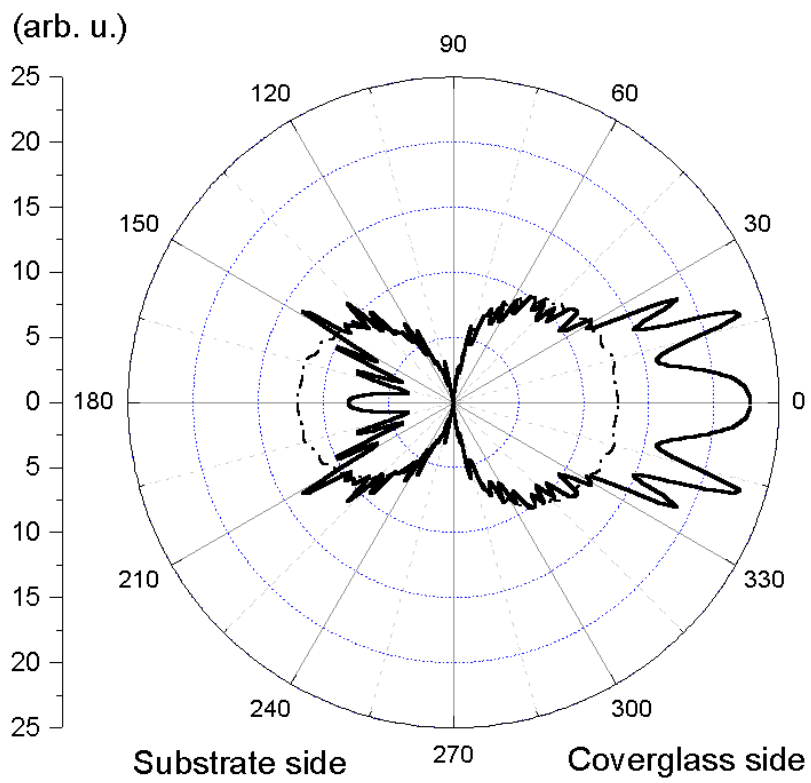

Fig. 8. Integrated radiation patterns of 3D-dipoles emitting at $680 \mathrm{~nm}$ and uniformly distributed in a $3 \mu \mathrm{m}$-thick layer lying at the top of the mirror (solid line) and on a bare silica substrate (broken line).

\section{Microcavity Experiments}

\section{Methods and Results}

In the microcavity experiment the dye used was Rhodamine 6G (R6G) dissolved in propylene carbonate. Propylene carbonate was chosen as the solvent because of its low intrinsic fluorescence and low volatility. R6G is highly 
efficient and can be excited with the $488 \mathrm{~nm}$ line from an argon ion laser.

The dye solution is placed in a microcavity consisting of two dielectric mirrors, made from alternating layers of silica $\left(n_{L}\right.$ $=1.5$ ) and Tantalum pentoxide $\left(n_{H}=2.26\right)$. The peak reflectivity of the mirrors was designed to be at $560 \mathrm{~nm}$. The top layer of each mirror is silica, deliberately grown $20 \mathrm{~nm}$ thinner than the $\lambda / 4 n$ condition. The microcavity is formed by placing a drop of the dye solution on one mirror, and then pressing the other mirror on top. Placing the cavity in a vacuum chamber for around an hour causes the liquid to slowly evaporate, pulling the two mirrors together. The resulting structure is a $\lambda / 2 n$ thick microcavity with a dye layer, approximately $40 \mathrm{~nm}$ thick, at the centre. In the microcavity case we can confirm that we have reached this minimum thickness by monitoring the evolution of the FabryPerot fringes formed by transmitting white light through the cavity.

The cavity material is the lower index silica so that the dye layer sits at the antinode of the electric field of the fundamental cavity mode, into which the molecules preferentially emit. This narrows the emission spectrum to match the cavity band- width and so increases the efficiency with which the light can be collected through the narrowband- pass filter used to discriminate the fluorescence from scattered laser light. The narrowing of the emission spectrum can be seen in figure 9 which shows the emission spectrum obtained from a microcavity (solid line) and from a sample that does not have the multilayer dielectric mirror coatings, but which is identical in all other respects (broken line).

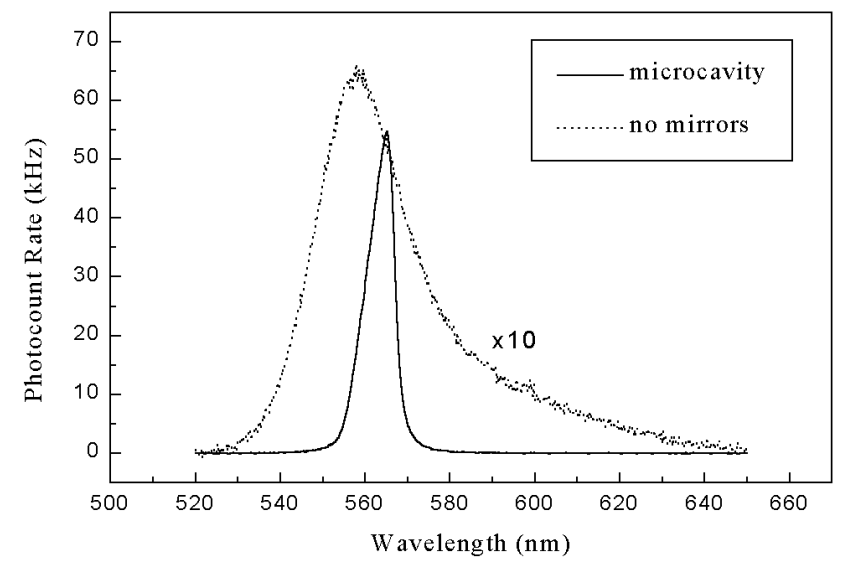

Fig. 9. Fluorescence spectrum of R6G in propylene carbonate measured in the microcavity (solid line) and in a structure without mirrors (broken line). The measurements for the sample without mirrors have been multiplied by a factor of 10 to improve clarity.

As well as narrowing the emission spectrum the microcavity also increases the total intensity of the emitted light by a factor of two, because the structure is designed to only emit light through the top mirror. This is achieved by designing the output mirror (with 5 pairs of layers) to have around 10\% transmission over the range $540-590 \mathrm{~nm}$, while the second mirror (with 9 pairs of layers) is highly reflecting (99.9\%). The increase in emitted light is evident from the measured spectra in figure 9 . The area under the curve for the microcavity sample is twice the area under the curve for the sample without the mirrors. We can estimate the geometric collection efficiency from the ratio of the solid angle subtended by our collecting lens in the silica mirror substrate compared with the full $2 \pi$ steradians that the dye emits into. For our lens of NA 0.35 we find a geometric collection efficiency of $2.5 \%$. We could increase this efficiency by increasing the numerical aperture of our collecting lens. However beyond NA 0.5 we would expect the cavity detuning due to off-axis light would begin to significantly broaden the spectrum of collected light.

The single molecule regime is reached by using very dilute $\left(10^{-9} \mathrm{M}\right)$ dye solutions and collecting light from a $6 \mu \mathrm{m}$ diameter spot using a confocal fluorescence microscope similar to that shown in figure 6 . The $488 \mathrm{~nm}$ light from a $\mathrm{CW}$ argon ion laser is focused through a $100 \mu \mathrm{m}$ pinhole and then through a microscope objective (x25, $0.35 \mathrm{NA}$ ) onto the microcavity. The collected light passes through the dichroic mirror and an interference filter $(580 \mathrm{~nm}, 10 \mathrm{~nm}$ bandwidth) removes the remaining laser light. A highly rejecting notch filter is not needed in our case, contrarily to the mirror experiment. The fluorescence light is divided equally between two avalanche diode single photon counting detectors which are connected to a photocount correlator and to a time interval analyser. Two detectors are used to circumvent the problems associated with the deadtime of the detectors $(1 \mu \mathrm{s})$, allowing the measurement of time intervals as small as $0.5 \mathrm{~ns}$.

When there are a small average number of dye molecules in the excitation volume the fluorescence signal exhibits strong fluctuations. The fastest fluctuations arise from the following mechanism: a dye molecule that has emitted a photon must remain dark for a time comparable to the excited state lifetime. This is commonly known as anti-bunching. On intermediate timescales we see fluctuations arising from passage through the triplet state to the ground state by non-radiative routes. On long timescales there are fluctuations in the number of dye molecules in the measurement volume due to diffusion in the liquid suspension.

We have shown [25] that for our system that

$$
\mathrm{g}^{(2)}(\tau)=1+\frac{1}{\mathrm{M}\left(1+\tau / \mathrm{t}_{\mathrm{d}}\right)}\left(1-(1+\mathrm{a}) \exp \left(-\tau / \mathrm{t}_{\mathrm{e}}\right)+\mathrm{a} \exp \left(-\tau / \mathrm{t}_{\mathrm{t}}\right)\right)
$$

where $t_{e}, t_{t}, t_{d}$ are the time constants related to the excited state lifetime, the triplet state lifetime and the diffusion time respectively. An interesting feature of this result is the zero time case; $g^{(2)}(0)=1$.

Each molecule emits antibunched light which if measured independently would show $g^{(2)}(0)=0$. However we 
measure an ensemble average from a fluctuating small number of molecules and as a result the measured $g^{(2)}(0)=1$ [25].

Figure 10 is a plot of $g^{(2)}(\tau)$ for time scales ranging from ns to seconds obtained for a cavity containing various concentration of dye solution with an incident laser power of $30 \mathrm{~mW}$ (measured at the sample). The solid lines are fits to equation [3]. The curves clearly show the three features that we expect. The initial rise due to antibunching, the drop at around $1 \mu$ s due to triplet state shelving and the drop at around $1 \mathrm{~ms}$ due to diffusion. We see that the number of molecules in the volume varies between $M=0.3-3$. The positive slope at zero delay in the correlation function is a clear sign that the emitted light is non-classical. The short triplet lifetime we measure is a result of triplet state quenching by dissolved oxygen with the subsequent creation of singlet oxygen. From experiments carried out at various laser powers we find clear evidence that the dye bleaches due to oxidisation by the highly reactive singlet oxygen. In ongoing experiments we are investigating dye performance in the presence of other triplet quenching agents in oxygen free solutions. We do see evidence of improved dye lifetime but as yet have not been able to freeze our system and study fixed single molecules.

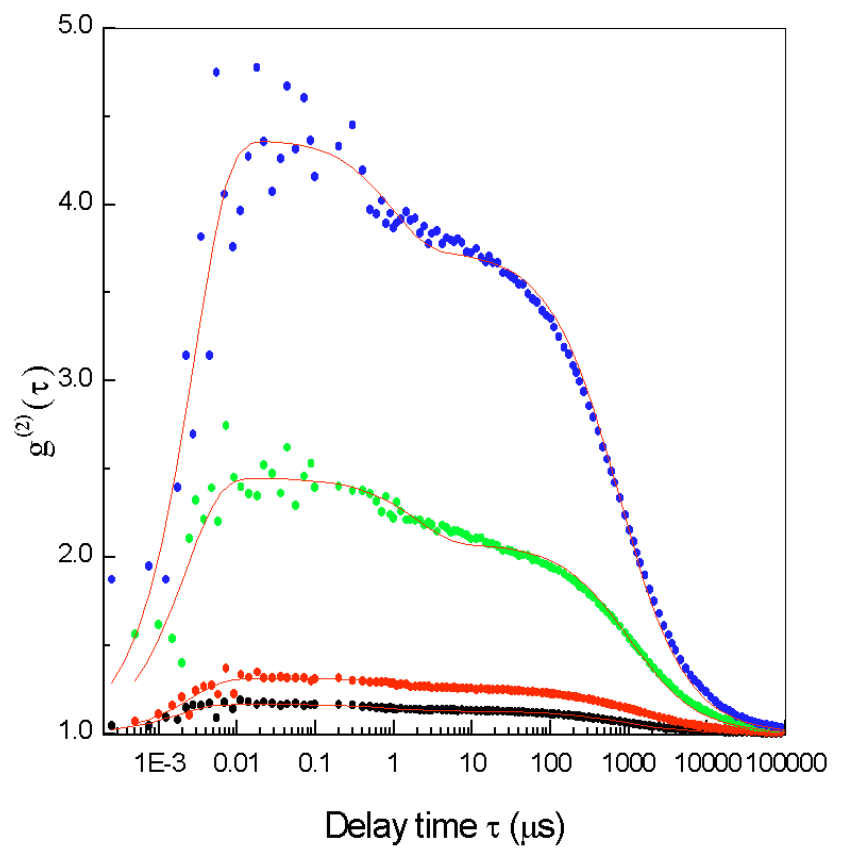

Fig. 10. $g^{(2)}(\tau)$ vs $\tau$ for a range of dye concentrations from $10^{9} \mathrm{M}$ to $10^{-8} \mathrm{M}$.

\section{Discussions}

From the number fluctuation results we have an estimate of the number of molecules in the volume while at the same time we are measuring the intensity. We find that in the case where triplet lifetimes are short due either to dissolved oxygen or triplet quenching agents such as cyclo-octatetraene (COT) the saturation photodetection rate is around 50 kilo-counts per second per molecule with negligible background scattered light $(<3 \mathrm{KHz})$. This low background level is remarkable, all the more as our pump power density is much higher than in the mirror experiment. When we consider that our geometric collection efficiency is only $2.5 \%$ and typical saturation emission rate for R6G will be of order $2.10^{7}$ per second, we find a combined filter transmission and detector efficiency of order $10 \%$. However we would like to reach a higher counting rates per molecule for rapid assay of lifetimes which in the simplest case would involve increasing the numerical aperture of our collection optics. This is limited in practice by the long working distance through the microcavity mirror. However there is also a theoretical limit to the amount we can increase the numerical aperture in the planar microcavity before the range of angles we access becomes enough to broaden the emission spectrum beyond the $10 \mathrm{~nm}$ filter width we are working with. As a rule of thumb we find that the collection efficiency and the range of wavelengths are both proportional to $1-\cos \theta$ where $\theta$ is the internal collection efficiency angle. For $10 \%$ geometric collection efficiency the range of wavelengths is also $10 \%$ so we would need to use a wider filter to collect all the microcavity emission and possibly cause a degradation of signal-to-background ratio.

\section{CONCLUSION}

The asset of planar dielectric microcavities for single molecule detection lies both on spatial and spectral modifications of spontaneous emission. Spatially, their ability to reorganize the radiation pattern can be used to concentrate fluorescence light in a privileged detection cone. This effect only holds for a limited spectral range and hence offers the possibility to use narrow filters to achieve high signal-to-background ratios. With our microcavity, a count rate as high as 50 kilo-counts per molecule has been obtained with R6G and low geometrical collection efficiency (2.5\%). This has enabled us to see clear evidence of photon antibunching on the nanosecond timescale. Thus, in the context of photophysical studies, the increase of detectivity offered by such a structure could be exploited to carefully monitor molecular states and determine very short kinetic parameters such as described in [26]. This is of course of particular interest for molecules whose quantum efficiency is lower than R6G. In this respect, the enhancement effect we observe for Cyanine 5 molecules on a simple mirror is encouraging. Larger increase is expected with higher and broader reflectivity. With a good rejection of the pump wavelength, very high NA collection optics could be used and eventually we may replace the bulk parabolic collection mirror such as used in [27]. Note that the planar geometry could be a real advantage in a number of biological situations, for screening devices or biochip fluorescence 


\section{Molecules RESEARCH PAPER}

analysis. Other planar designs are possible to exert spontaneous emission control on emitters in liquid solutions, and previous studies have shown that mono- or bi-periodic diffraction gratings are very promising candidates [28, 29].

Acknowledgement The authors acknowledge the help of S.C. Kitson in the development of the technique. They are grateful to $\mathrm{C}$. Picard and $\mathrm{H}$. Bazin for the Europium chelate and Cyanine 5 molecules used in this work C.B. is supported by a grant of the French Ministry of Defence.

\section{References}

[1] For a review see: Nie, S., Zare R.N. Annu. Rev. Biophys. Biomol. Struct. 26 (1997) 567

[2] Mets Ü, Rigler R. J. of Fluoresc. 4 (1994) 259

[3] Nie S, Chiu DT, Zare RN. Science 266 (1994) 1018

[4] Betzig, E., Chichester R.J. Science 262 (1993) 1422

[5] Ambrose P.W., Goodwin P.M., Martin J.C., Keller R.A. Phys. Rev. Lett. 72 (1994) 160

[6] Purcell E.M., Phys. Rev. 69 (1946) 681

[7] Drexhage K.H. In: Progress in Optics XII. Wolf E. editor. North-Holland, Amsterdam (1974) 163

[8] Goy P., Raimond J.M., Gross M., Haroche S. Phys. Rev. Lett. 50 (1983) 1903

[9] Yamamoto Y., Slusher R.E. Phys. Today 46 (1993) 66

[10] For a recent review of the field see: Benisty H., Gérard J.-M., Houdré R., Rarity J.G., Weisbuch C., editors. Confined photon systems: fundamentals and applications, Springer, Berlin (1999)

[11] Gérard J.M., Sermage B., Gayral B., Legrand B., Costard E., Thierry-Mieg V. Phys. Rev. Lett. 81 (1998) 1110
[12] Macleod H.A. Thin film Optical Filters. Adam Hilger Ltd, Bristol (1986) 236

[13] Björk G., Machida S., Yamamoto Y., Igeta K. Phys. Rev. A 44 (1991) 669

[14] Yamamoto Y., Machida S., Björk G. Phys. Rev. A 44 (1991) 657

[15] DeMartini F., Innocenti G., Jacobovitz G.R., Mataloni P. Phys. Rev. Lett. 59 (1987) 2955

[16] Rigneault H., Monneret S. Phys. Rev. A 54 (1996) 2356

[17] Rigneault H., Robert S., Begon C., Jacquier B., Moretti P. Phys. Rev. A 55 (1997) 1497-1502

[18] Benisty H., De Neve H., Weisbuch C. IEEE J. Quant. Electron. 34 (1998) 1612 and Benisty H., De Neve H., Weisbuch C. IEEE J. Quant. Electron. 34 (1998) 1632

[19] Galaup C., Picard C., Cazaux L., Tisnès P., Aspe D., Autiero H. New J. Chem. 20 (1996) 997

[20] Rigneault H., Monneret S., Westbrook C. J. Opt. Soc. Am. B 15 (1998) 2712

[21] Rigneault H., Amra C., Begon C., Cathelinaud M., Picard C. Appl. Opt. 38 (1999) 3602

[22] Rigler R., Mets Ü., Widengren J., Kask P. Eur. Biophys. J. 22 (1993) 169

[23] Schwille P., Bieschke J., Oehlenschläger F. Biophys. Chem. 66 (1997) 211

[24] Koppel D.E. Phys. Rev. A 10 (1974) 1938

[25] Kitson S.C., Jonsson P., Rarity J.G., Tapster P.R. Phys. Rev. A 58 (1998) 620

[26] Eggeling C., Fries J.R., Brand L., Günther R., Seidel C.A.M. Proc. Natl. Acad. Sci. USA 95 (1998) 1556

[27] Brunel C., Lounis B., Tamarat P., Orrit M. Phys. Rev. Lett. 83 (1999) 2722

[28] Kitson S.C., Barnes W.L., Sambles J.R. Opt. Comm. 122 (1996) 147

[29] Rigneault H., Lemarchand F., Sentenac A., Giovannini H. Opt. Lett. 24 (1999) 148 